\title{
Stereo-PIV study of flow around a maneuvering fish
}

\author{
by \\ J.Sakakibara, M.Nakagawa ${ }^{1}$ and M.Yoshida ${ }^{2}$ \\ Institute of Engineering Mechanics and Systems, University of Tsukuba, Tsukuba, \\ 305-8573, Japan
}

\begin{abstract}
Stereoscopic PIV was used to measure the three-component velocity distribution around a live fish. At the same time, shadow images of the fish were captured by additional cameras in order to reconstruct the three-dimensional shape of the fish. The side jet shed against the swept tail during turning was evident in the results. The height of the jet was approximately equal to the height of the tail fin, and vortexs ring were observed around the side jet. Based on the circulation and diameter of the vortex ring, the impulse and time-averaged force in terms of the side jet were estimated.
\end{abstract}

\footnotetext{
${ }^{1}$ Present address: Toyoda Machine Works Ltd., Aichi, Japan

${ }^{2}$ Present address: Fuji Heavy Industries Ltd., Japan
} 


\section{Introduction}

The propulsion of fishes has been extensively investigated over the past century. Breder (1926) systematized the body movements of swimming fish according to several categories, such as anguilliform motion, in which the head sways as much as the tail, and carangiform motion, in which the head sways slightly compared to the tail. Although Breder's work adopted a zoological point of view, it also considered specific applications of fish locomotion to underwater vehicles. Gray (1936) estimated the propulsive efficiency of the dolphin and concluded that the dolphin's muscles can generate only one-seventh of the power needed for it to swim. This conclusion, known as Gray's paradox, was followed by several theoretical works based on fluid mechanics. Years later, Lighthill (1960) predicted the propulsive efficiency of the anguilliform motion by using the slender body theory.

While theoretical study based on the non-viscous potential flow theory has been investigated extensively by Lighthill(1970) and Wu(1971), several investigators have studied the vortex dynamics of a flow induced by the unsteady motion of a body in a viscous fluid. Taneda and Tomonari (1974) investigated the flow around a flexible plate performing a swimming motion and found that the swimming motion accelerates the flow near the surface in the wave direction, reduces the boundary layer thickness, and suppresses the turbulent fluctuation. Gopalkrishnan et al.(1994) revealed details of vortex dynamics and optimal control parameters of an oscillating foil placed in the wake of a cylinder. Anderson et al. (1998) performed force and power measurements of an oscillating foil in a uniform flow. They showed that the propulsive efficiency was as high as $87 \%$ under the condition of optimal wake formation.

Recently, a direct measurement of the flow around live swimming fish was achieved 
by using two-component particle image velocimetry (PIV) in order to resolve the velocity field in a horizontal plane. Stamhuis and Videler (1995) first reported PIV and PTV measurements of flow around live aquatic animals such as copepods or mullets. Muller et al. (1997) analyzed the structure of the wake behind a continuously swimming mullet by using PIV, showing a wake consisting of a zigzagging jet flow between alternating vortices, and estimated thrust energy and power based on the measured velocity data. They found that the undulatory oscillation of the body causes less than half of the energy shed in the wake and that the remainder was generated by the tail. Wolfgang et al. (1999a) observed the vortex shedding that occurs during straight-line swimming and turning, and suggested that the wake vorticity is generated upstream of the tail by the undulations of the body and that it merges with the vorticity shed at the trailing edge of the tail.

While their observations shed light on our understanding of the propulsion mechanism of fish, the flow observed by them was not three-dimensional and may have included variations in the direction of the fish body height. The importance of role of the three-dimensional flow field near the fish body was recognized by the three-dimensional numerical results of the flow around swimming fish presented by Wolfgang et al. (1999b),

Experimental efforts to understand three-dimensional flow structures have been continued by Drucker and Lauder (1999, 2000, 2001), who reconstructed the three-dimensional wake structures around the pectoral fins of steadily-swimming bluegill sunfish. They measured the velocity distribution in three orthogonal planes behind the fin by using PIV, and found two distinct vortex rings linked ventrally. The wake momentum and time averaged force exerted by the fin stroke was estimated based on the vortex ring circulation calculated from the measured velocity data. While their study revealed much of the flow structures of the pectoral fin wake, experimental evidence of the three-dimensional flow 
induced by the tail fin of live fish has not been reported so far.

This paper aimed to visualize the flow induced by the tail fin of a live fish, especially focusing on turning motion. First, ordinary two-component PIV was employed to reveal the horizontal flow field around the fish during turning, as in Wolfgang et al. (1999a). Then, stereoscopic PIV, which resolves the instantaneous out-of-plane velocity component in addition to the in-plane component, was used to measure the three-component velocity in a vertical plane behind the fish during turning and a horizontal plane around the fish during successive turns.

Simultaneous with the stereoscopic PIV measurement, the three-dimensional shape identification technique was applied to estimate the location of the fish relative to the measuring plane. Finally, we estimated the shape of the vortex structures, and the impulse and thrust force of the maneuvering fish.

\section{Materials and Methods}

\subsection{Experimental Apparatus and Instruments}

Experiments were conducted in a Plexiglas tank having a length of $1000 \mathrm{~mm}$, a span of $200 \mathrm{~mm}$ and a water depth of $300 \mathrm{~mm}$ (Fig.1). A goldfish (110mm long from head to tail) was released in this tank and allowed to swim freely. The fish was given no external stimulation to the fish to control its motion. We just waited until the fish entered the region of view and performed turning motions without prompting. Ambient flow conditions just before the measurements were not controlled. Thus the velocity data were carefully examined in order to avoid the inclusion of the effects of apparent flow structures created by actions performed prior to the measurements. 
The water temperature was maintained above $20^{\circ} \mathrm{C}$ to make the fish swim actively. Note that goldfish swim in a carangiform manner, i.e., the head sways much less than the tail fin.

A 2-mm-thick laser light sheet produced by a Nd-YAG laser (New Wave Research, Minilase PIV-30) equipped with a laser delivery arm (house-built) illuminated the flow field. To minimize the potentially disturbing effects of the strong laser light, the light was turned on only when the fish entered the measurement region. In the case of stereoscopic PIV measurement in a vertical plane, two CCD cameras (Kodak, ES1.0), referred to as CCD cameras 1 and 2 in Fig.1, were placed at both sides of the tank, and viewed the same region of interest in the light sheet plane. The angle between the optical axes of the two cameras was set at about 90 degrees, and lenses were mounted to satisfy the Scheimpflug condition. Prisms, which were made from Plexiglas containers filled with water, were placed outside the tank and used to make the optical axis perpendicular to the air/tank interface. Without this step, the images would not be well focused due to the different refraction angle between meridional rays and sagittal rays (see Hecht, 1990) striking the lens from the object. In the case of velocity measurements in the horizontal plane, the CCD cameras were placed under the tank. Here, for the stereoscopic PIV measurements, the prism were placed under the tank, and the angle between the optical axes of the two CCD cameras was set at about 90 degrees. For two-component PIV measurements (see Sec.3.1), CCD cameras were arranged to make the optical axis perpendicular to the light sheet plane illuminating the horizontal plane.

The images captured by these CCD cameras were stored in the host memory of a $\mathrm{PC}$ via image grabbers (Imaging Technology, IC/PCI) with a frame rate of $30 \mathrm{fps}$. Our PIV system could typically capture 150 successive time-dependent images from both cam- 
eras. Since an instantaneous velocity field was measured based on the double images, 75 instantaneous velocity maps with $1 / 15$ s intervals were obtained by a cross-correlationbased two-component PIV algorithm or a stereoscopic PIV algorithm as described in the following section.

In order to identify the shape and location of the fish, we used additional light sources and cameras: two conventional CCD cameras (Sony, XC75), referred to as CCD cameras 3 and 4 in Fig.1, and strobe lights (Nissin electronic, MM-10). A sheet of white paper was placed between each strobe lamp and the Plexiglas tank to diffuse the light. One camera viewed from the bottom and the other from the side. Each CCD camera faced the strobe light placed on the opposite side of the Plexiglas tank. The images were captured by dual frame grabbers (Data Translation, DT3155) in the other PC and stored in the host memory. The algorithm for the shape identification is described in Sec.2.3.

\subsection{Stereo-PIV Algorithm}

In this study, stereoscopic PIV technique, which is capable of resolving time-dependent three-component velocity in a two-dimensional slice, was employed to measure the velocity field. This method requires two cameras viewing the same region in a measurement plane from different directions, and measures the out-of-plane displacement of the particles in a stereoscopic sense in the manner of a human eye. Prasad and Adrian (1993), who first presented this technique as fluid velocity measurements, calculated the three-dimensional particle displacement from the geometrical intersection of two projection lines from particles to the image planes. The image distortion caused by the aberrations of the optical system or various magnifications in the image plane was corrected by a technique based on a mathematical model of the distortion. In order to decrease the error due to the 
distortion, a generalized stereoscopic PIV method was established by Solof et al.(1997). Their method requires experimental position calibrations in order to obtain the mapping functions between physical coordinate and image coordinate. After obtaining the mapping functions, the displacement of particle image is equated by product of a tensor consisting of partial derivative of the mapping functions respective to the physical coordinate and displacement vector of physical particle. By solving the equations, physical particles displacement is obtained. This method does not require any geometrical calculation for obtaining an intersection of two projection lines. If the mapping function is simple polynomial and is not based on any physical model such as the pin-hole camera model, it is called 'direct mapping' method.

Our house-build stereoscopic PIV system is based on a new algorithm which is rather similar to the older method of Prasad and Adrian(1993) in terms of the geometrical consideration of particle displacement, but we used experimental position calibration in order to allow optical distortion of the images. The mapping function we used in this study was of 'direct mapping'. Figure 2 shows the geometrical relationship between a particle inside the laser light sheet and its image on the CCD surface of two cameras viewing approximately the same area from different directions. Here, the $x$ - and $y$-axes in the physical three-dimensional coordinate system are parallel to the laser light sheet, and the $z$-axis is normal to the sheet. A particle, or a volume containing multiple particles, located at given point $\boldsymbol{x}$ in the physical coordinate system was imaged at $\boldsymbol{X}^{L}\left(\boldsymbol{X}^{R}\right)$ in the two-dimensional image coordinates of the camera $\mathrm{CCD}^{L}\left(\mathrm{CCD}^{R}\right)$. Here and in the following, the superscripts $L$ and $R$ denote the quantity relating to the "left" and "right" cameras. The above relationships can be expressed using mapping functions, $\boldsymbol{F}_{0}^{L}\left(\boldsymbol{F}_{0}^{R}\right)$, 
from the physical coordinate at $z=z_{0}$ to the image coordinate as follows:

$$
\begin{aligned}
& \boldsymbol{X}^{L}=\boldsymbol{F}_{0}^{L}(\boldsymbol{x}) \\
& \boldsymbol{X}^{R}=\boldsymbol{F}_{0}^{R}(\boldsymbol{x})
\end{aligned}
$$

After a short period of time $\Delta t$, the particle moves to $\boldsymbol{x}+\Delta \boldsymbol{x}$, and its images displace at $\boldsymbol{X}^{L}+\Delta \boldsymbol{X}^{L}\left(\boldsymbol{X}^{R}+\Delta \boldsymbol{X}^{R}\right)$ in the image coordinate. Now consider a light path extending from the particle position $\boldsymbol{x}+\Delta \boldsymbol{x}$ to the position $\boldsymbol{X}^{L}+\Delta \boldsymbol{X}^{L}\left(\boldsymbol{X}^{R}+\Delta \boldsymbol{X}^{R}\right.$ ). These lines come across the plane $z=z_{0}$ at $\boldsymbol{x}_{0}^{L}\left(\boldsymbol{x}_{0}^{R}\right)$. While the light path might be bent at, for example, the air-water interface of the experimental apparatus, or might be curved due to aberrations in the lens system, the light still travels straight in a uniform medium of constant density (or temperature), such as an isothermal flow field. If the planes $z=z_{0}$ and $z=z_{1}$ are both inside such a medium, the light path from $\boldsymbol{x}_{0}^{L}\left(\boldsymbol{x}_{0}^{R}\right)$ to the particle is extrapolated linearly to $\boldsymbol{x}_{1}^{L}\left(\boldsymbol{x}_{1}^{R}\right)$ in the plane $z=z_{1}$. Thus, the particle location $\boldsymbol{x}+\Delta \boldsymbol{x}$ is determined as the intersection of the lines $\boldsymbol{x}_{0}^{L} \rightarrow \boldsymbol{x}_{1}^{L}$ and $\boldsymbol{x}_{0}^{R} \rightarrow \boldsymbol{x}_{1}^{R}$. Using mapping functions from the image coordinate to the physical coordinate at $z=z_{0}$, $\boldsymbol{f}_{0}^{L}\left(\boldsymbol{f}_{0}^{R}\right)$, and at $z=z_{1}, \boldsymbol{f}_{1}^{L}\left(\boldsymbol{f}_{1}^{R}\right)$, the above four points are represented as

$$
\begin{gathered}
\boldsymbol{x}_{0}^{L}=\boldsymbol{f}_{0}^{L}\left(\boldsymbol{X}^{L}+\Delta \boldsymbol{X}^{L}\right) \\
\boldsymbol{x}_{1}^{L}=\boldsymbol{f}_{1}^{L}\left(\boldsymbol{X}^{L}+\Delta \boldsymbol{X}^{L}\right) \\
\boldsymbol{x}_{0}^{R}=\boldsymbol{f}_{0}^{R}\left(\boldsymbol{X}^{R}+\Delta \boldsymbol{X}^{R}\right) \\
\boldsymbol{x}_{1}^{R}=\boldsymbol{f}_{1}^{R}\left(\boldsymbol{X}^{R}+\Delta \boldsymbol{X}^{R}\right) .
\end{gathered}
$$

Since the intersection of two lines may not exist due to errors in the above mapping functions or in the particle displacement, we define the "intersection" as the center of the smallest sphere to which both lines are tangential. Consider the case in which two lines 
$\boldsymbol{x}_{0}^{L} \rightarrow \boldsymbol{x}_{1}^{L}$ and $\boldsymbol{x}_{0}^{R} \rightarrow \boldsymbol{x}_{1}^{R}$ are respectively tangential to the sphere at a node A, $\boldsymbol{x}_{0}^{L}+\alpha \boldsymbol{n}^{L}$ , and a node B, $\boldsymbol{x}_{0}^{R}+\gamma \boldsymbol{n}^{R}$ as illustrated in Fig.3.

Here the $\alpha$ and $\beta$ are scalar, and $\boldsymbol{n}^{L}$ and $\boldsymbol{n}^{R}$ are unit vectors written as

$$
\begin{aligned}
\boldsymbol{n}^{L} & =\frac{\boldsymbol{x}_{1}^{L}-\boldsymbol{x}_{0}^{L}}{\left|\boldsymbol{x}_{1}^{L}-\boldsymbol{x}_{0}^{L}\right|} \\
\boldsymbol{n}^{R} & =\frac{\boldsymbol{x}_{1}^{R}-\boldsymbol{x}_{0}^{R}}{\left|\boldsymbol{x}_{1}^{R}-\boldsymbol{x}_{0}^{R}\right|} .
\end{aligned}
$$

By defining a unit vector directed from node A to node B as $\boldsymbol{m}$ and the distance between these two nodes as $\beta$, the node $\mathrm{B}$ can be determined as

$$
\boldsymbol{x}_{0}^{L}+\alpha \boldsymbol{n}^{L}+\beta \boldsymbol{m}=\boldsymbol{x}_{0}^{R}+\gamma \boldsymbol{n}^{R} .
$$

Since the two lines $\boldsymbol{x}_{0}^{L} \rightarrow \boldsymbol{x}_{1}^{L}$ and $\boldsymbol{x}_{0}^{R} \rightarrow \boldsymbol{x}_{1}^{R}$ are perpendicular to line AB, the unit vector $\boldsymbol{m}$ is denoted as

$$
\boldsymbol{m}=\boldsymbol{n}^{L} \times \boldsymbol{n}^{R} .
$$

Thus, the three remaining unknowns $\alpha, \beta$ and $\gamma$ in eq.9 can be obtained by solving the matrix expression of the eq.9:

$$
\left[\begin{array}{c}
\alpha \\
\beta \\
\gamma
\end{array}\right]=\left[\begin{array}{c}
x_{0}^{R}-x_{0}^{L} \\
y_{0}^{R}-y_{0}^{L} \\
0
\end{array}\right]\left[\begin{array}{ccc}
n_{x}^{L} & m_{x} & -n_{x}^{R} \\
n_{y}^{L} & m_{y} & -n_{y}^{R} \\
n_{z}^{L} & m_{z} & -n_{z}^{R}
\end{array}\right]^{-1} .
$$

Finally, the point of intersection, which therefore is assumed to be the location to which the particle is displaced, can be determined as

$$
\boldsymbol{x}+\Delta \boldsymbol{x} \approx \alpha \boldsymbol{n}^{L}+\frac{\beta \boldsymbol{m}}{2} .
$$

The $\beta$ value represents the distance between two lines, which should be zero in an ideal case. Thus, the $\beta$ value could be used as a measure of the error in the three-dimensional 
displacement of particles in the interrogation region. This is a useful point of our algorithm. We provide a typical $\beta$ value for our measurement in Sec.2.5. Another major point of our algorithm is that only two different planes need to be calibrated in principle because the mapping function does not contain any $z$ related terms. Conventional stereoscopic PIV systems also require two planes for calibration, since their mapping function has only the first order $z$ terms by neglecting $z^{2}$ or higher order terms. The reason why they neglect higher order terms is that the variation of the particle position in the $z$ direction is expected to be much smaller than in other directions. However, finite error due to the linear approximation still may be remained, while our algorithm is free from this error. Inclusion of higher order terms, such as the function having $z^{2}$ terms given by Solof et al., reduces the error. But in this case, three different planes need to be calibrated that requires more calibration cost than ours.

\subsection{Identification of Body Shape}

In addition to the velocity measurement performed by PIV, it was necessary to definethe three-dimensional surfaces of the fish's body-fluid interface. Since the fish was moving, measurement of the exact three-dimensional surfaces of the body required a fairly high level of performance on the part of the instrumentation. Rather than measuring the exact shape, we obtained the approximate shape of the body without a loss of information needed to discuss the flow dynamics as described below.

All the images viewed from cameras 3 and 4 (Fig.1) were bright due to the light coming from the strobe lamp, except when the fish appeared within the region of interest. When the fish was inside this region, the projection of its body appeared as a shadowed region in the image. After thresholding this image at a certain threshold level, the image was 
scanned to identify the location of each pixel element composing the shadowed region; these elements were denoted as $\boldsymbol{X}^{S}$ and $\boldsymbol{X}^{B}$. Here, the superscript $S$ and $B$ represent the images taken by the camera at the "side" and "bottom", respectively.

We now define the physical coordinate system illustrated in Fig.4, as defined by the camera at the side viewing the $x-z$ plane, and the camera at the bottom viewing the $x-y$ plane. The mapping functions from the image coordinate $\boldsymbol{X}^{S}$ to the physical coordinate on the planes at $y=y_{0}$ and $y=y_{1}$ were defined as $\boldsymbol{f}_{0}^{S}$ and $\boldsymbol{f}_{1}^{S}$, respectively. The other mapping functions $\boldsymbol{f}_{0}^{B}$ and $\boldsymbol{f}_{1}^{B}$ are the same except from the image coordinate $\boldsymbol{X}^{B}$ to the physical coordinate on the planes at $z=z_{0}$ and $z=z_{1}$. These functions are the same type of polynomials used in the stereo-PIV method described in sec.2.2, and are estimated by means of the calibration procedure described in Sec.2.4. Using the above mapping functions, a ray through the image plane $\boldsymbol{X}^{S}$ passes through the physical points $\boldsymbol{f}_{0}^{S}\left(\boldsymbol{X}^{S}\right)$ on a plane at $y=y_{0}$ and $\boldsymbol{f}_{1}^{S}\left(\boldsymbol{X}^{S}\right)$ on a plane at $y=y_{1}$. This ray could be "drawn" in a virtual space such as a three-dimensional array consisting of $100 \times 100 \times 100$ of "0" initialized byte-type elements prepared in a computer, by filling " 1 " for the array elements on the ray. By repeating this for all the pixels of the shadowed region, the flux of the rays from the side can be established as illustrated in Fig.5(a).

The same operations for the ray through a point in the image plane $\boldsymbol{X}^{B}$ and physical points $\boldsymbol{f}_{0}^{B}\left(\boldsymbol{X}^{B}\right)$ and $\boldsymbol{f}_{1}^{B}\left(\boldsymbol{X}^{B}\right)$ on planes at $z=z_{0}$ and $z=z_{1}$ could be performed as shown in Fig.5(b). By performing a logical-AND operation between the two arrays (a) and (b) of Fig.5, the shape of the fish can be constructed as shown in Fig.5(c).

Although the shape estimated by this method does not resolve the individual motion of the fins, such as the pectoral fins and dorsal fins, it can still represent the horizontal/vertical movement of the body or the undulation of the tail fin. If the number of 
projected images can be increased by using additional cameras, the resolution of the three-dimensional shape will be improved.

\subsection{Calibration}

The mapping functions used in Sec.2.2 and Sec.2.3 were obtained by means of the following procedure.

Three calibration plates, one for PIV and other two for body identification, were set perpendicular to each other, such that the whole set of the plates could be accurately shifted in the direction normal to each surface by a motorized traversing mechanism. Each calibration plate has equally-spaced grids at $2 \mathrm{~mm}$ intervals, and a dot was drawn at the center of plate as a reference point. The relative position of each reference point was measured prior to the calibration.

First, the calibration plates were placed in a measurement volume, and the laser light sheet was adjusted on a plate that was parallel to the $y-z$ plane. After adjusting the CCD cameras for PIV to view this plate, the other cameras ( i.e., those for body identification) were adjusted so as to view the other plates that were respectively parallel to the $x-z$ and $x-y$ planes. The images captured by all cameras could be used to establish the

functions $\boldsymbol{F}_{0}^{L}, \boldsymbol{F}_{0}^{R}, \boldsymbol{f}_{0}^{L}, \boldsymbol{f}_{0}^{R}, \boldsymbol{f}_{0}^{S}$ and $\boldsymbol{f}_{0}^{B}$. The set of plates was then shifted $\Delta x$ towards the $x$-direction, which is normal to the PIV measurement plane. The value of $\Delta x$ was chosen to be $\Delta x=2 m m$, which was approximately 14 pixels of the displacement in the image. Since the error involved in determining the location of the grids by pattern matching, to be described below, was typically 0.1 pixels, the relative error was $0.1 / 14=0.7 \%$, which was sufficiently small for this measurement.

The images captured by the PIV cameras were used to derive the functions $\boldsymbol{f}_{1}^{L}$ and 
$\boldsymbol{f}_{1}^{R}$. After returning the set of plates to the original position, the plates were shifted $10 \mathrm{~mm}$ toward the $y$ - or $z$ - direction in order to capture the images for $\boldsymbol{f}_{1}^{S}$ or $\boldsymbol{f}_{1}^{B}$.

The images obtained above were processed individually by means of the following algorithms. Each grid point was searched by pattern matching methods, which found the peak location of the correlation of the original image and template pattern similar to the grid. After finding the location of each grid in the image coordinate, the physical location of each grid was determined automatically based on the location of the reference grid and known spacing of the grids. This procedure produced a list of image coordinates and physical coordinates of the grid points, and the least-square method was applied to calculate the coefficient of the third-order polynomials for the mapping functions, for example:

$$
\begin{aligned}
F_{0, X}^{L}(x, y)= & a_{1}+a_{2} x+a_{3} x^{2}+a_{4} x^{3}+a_{5} y+a_{6} x y+a_{7} x^{2} y \\
& +a_{8} y^{2}+a_{9} x y^{2}+a_{10} y^{3} \\
F_{0, Y}^{L}(x, y)= & b_{1}+b_{2} x+b_{3} x^{2}+b_{4} x^{3}+b_{5} y+b_{6} x y+b_{7} x^{2} y \\
& +b_{8} y^{2}+b_{9} x y^{2}+b_{10} y^{3} .
\end{aligned}
$$

Note that these functions are independent of the variable $z$, because the function is limited in a fixed $z$ location.

\subsection{Interrogation and Errors}

For the two-component PIV measurement in a horizontal plane, the size of the measurement region was approximately $200 \times 200 \mathrm{~mm}^{2}$ and the interrogation spot size was $6 \times 6 \mathrm{~mm}^{2}$. The interval of measurement points was $5 \mathrm{~mm}$ in both the $x$ and $y$ directions. The uncertainty of velocity measurement was $2.5 \mathrm{~mm} / \mathrm{s}$ in both the $u$ and $v$ components. 
For the stereoscopic PIV measurement in a vertical plane, on the other hand, the measurement region was $140 \times 100 \mathrm{~mm}^{2}$ and the interrogation spot size was $4.2 \times 3 \mathrm{~mm}^{2}$. The interval was $2 \mathrm{~mm}$ in both the $y$ and $z$ directions. The uncertainty of the velocity measurement was estimated by assuming the subpixel accuracy of the particle displacement measurement as 0.1 pixel. The estimated uncertainty was $1.8 \mathrm{~mm} / \mathrm{s}$ in the $v$ component, and $2.5 \mathrm{~mm} / \mathrm{s}$ in both the $u$ and $w$ components. As mentioned previously, the $\beta$ value is an indicator of the error associated with particle displacement measurement. Unfortunately, we did not record the $\beta$ value in the present study. Instead, we provide the $\beta$ value measured by the same PIV system in alternate experiments, which measured the velocity distribution in a cross-section of the cylinder wake formed in a uniform flow having spatial and time scales similar to the present study. The standard deviation of $\beta / \Delta t$ was approximately $0.6 \mathrm{~mm} / \mathrm{s}$ in a uniform flow, and $6 \mathrm{~mm} / \mathrm{s}$ in the shear layer.

\section{Results and Discussion}

\subsection{Single Turning}

This section discusses the flow around a fish making a single turning motion. Timedependent velocity vector maps in a horizontal plane at the mid height of the fish body, measured by the ordinary two-component PIV, are shown in Fig.6 superposed by the iso-vorticity contours and the body shape.

Initially the fish body was in a straight form at $t=0$ s (a). Suddenly, however, at $t=1 / 15 \mathrm{~s}(\mathrm{~b})$, the fish bent its body in the middle. Here the flow towards the body was observed in the gulf of the bending body. As reported by Wolfgang et al.(1999a), the vorticity is already generated on the surface of the body. After this moment, the bending 
point was propagated towards the tail and the counter-rotating vortex pair represented by two maximums of vorticity was formed just behind the tail fin (c). At the middle of the counter-rotating vortices, a flow toward the negative $y$ direction can be observed. This flow, referred to as a "side jet" issuing toward the angular direction of the turning fish, was expected to produce an angular momentum of the fish body and its added mass. This is very much like a sprinkler, which issues a water jet in the angular direction and gains angular momentum.

At $t=8 / 15 \mathrm{~s}(\mathrm{~d})$, the body becomes straight again and the fish swims away in a forward direction. The direction of the body was rotated approximately 45 degrees in the entire sequence above. The counter-rotating vortices were separated from the swept tail fin and slightly convected downstream. The contour of positive vorticity was elongated toward the positive $y$ direction and showed another maximum of the vorticity near the tail. Between this vorticity maximum and the tip of the tail fin, another jet toward the positive $y$ direction was formed. This jet, designated a "thrust jet", produces a thrust force that acts on the fish during its forward movement. The thrust jet was originally observed by the Wolfgang et al.(1999a).

The side jet and thrust jet were also evident in the following stereoscopic PIV results. Figure 7 shows the three-component velocity in the $y-z$ plane and the three-dimensional body shape at successive instants. In the figures at the left, black arrows represent the $v$ and $w$ components of velocity, and the color intensity of red (blue) represents the magnitude of the negative (positive) $u$ component velocity. In the figures in the right column, the data is identical to that in the left column but the view is from the top. The $u$ and $v$ components of the velocity vectors are indicated by arrows. The vectors and color scales are erased where the laser light sheet is interrupted by some part of the fish body, 
or if the light scattered from the particle could not reach one of two CCD cameras for the same reason.

The fish body was initially straight at $t=0 \mathrm{~s}(\mathrm{a})$, and the tail fin cut across the PIV measurement plane $(y-z$ plane $)$. Then, at $t=7 / 15 \mathrm{~s}(\mathrm{~b})$, the body was bent and the tail fin became parallel to the $y-z$ plane. Note that the velocity vectors near the tail fin were deleted because the laser light sheet was interrupted by the tail fin, or because the tail fin hid the tracer particles at the right side (left side) of the fins from the camera at the left side (right side), although the particles had to be observed by both cameras.

In the next instant at $t=16 / 15 \mathrm{~s}(\mathrm{c})$, the tail was returned to its original position and the body became straight. Only a tip of the tail fin remained in the $y-z$ plane. At the right side of the tail fin, a jet toward the lower right corner is visible within the blue-colored region. The velocity vectors of the jet are actually directed normal to the body, as shown in the figure in the right column. Since the direction and the timing of the formation of the jet are quite similar to those for the side jet observed in Fig.6, we believe this jet is the side jet producing the angular momentum of the body. Although the rotation angle of the fish was approximately 24 degrees, which was smaller than the angle found in Fig.6, the qualitative features of the flow pattern may not have been altered significantly. In the vicinity of the tail, a strong backward flow (negative $x$ direction, colored red) was produced. This is apparently the thrust jet observed in Fig.6. Both the side jet and the thrust jet, respectively identified as the blue region and red region in the figure, have approximately the same height as the tail fin, and both jets were accompanied at top and bottom by counter-rotating vortices. Since we already observed counter-rotating vortices beside the side jet in a horizontal plane as shown in Fig.6, the side jet could be surrounded by a vortex ring, if both the counter-rotating vortex pairs in 
the horizontal and the vertical plane were connected in a circle. A schematic drawing of the arrangement of vortex ring and the side jet are shown in Fig.8.

The next Figure (Fig.7(d)), at $t=16 / 15 \mathrm{~s}$, had a flow pattern similar to that observed in Fig.7(c), but the body proceeded forward and the tail fin was moved away from the $y-z$ plane. The side jet was convected toward the lower right corner, as was also shown in Fig.6.

\subsection{Double Turning}

Figure 9 shows successive maps of three-component velocity vectors measured in a horizontal plane through the fish which makes two turns in a short period. Figures 9(a)-(f) are viewed from the top, and Fig.9( $\left.\mathrm{c}^{\prime}\right)$ and $\left(\mathrm{f}^{\prime}\right)$ are from the back of the fish at the same instants as those of (c) and (f). Vectors and color scale represents the in-plane and out-of-plane velocity components, respectively.

In Fig.9(a) the fish is in a straight form without any propulsive jet. Then, the fish suddenly bent its body in Fig.9(b). After 3/15s, the tail was swept back and the body rotated in a clockwise direction. On the right side of the tail, the side jet, which is in the middle of counter rotating vortices, is ejected upper-right direction (in this figure), which is approximately normal to the body axis. Here, the measurement plane is located slightly above the mid-height of the body, as shown in Fig.9(c') which is a side view from the back of the body. The color scale shows that the upstream and downstream of the center of the counter rotating vortices has a large magnitude of out-of-plane velocity vector component. In order to see the out-of-plane motion of the fluid in this part, projection of the vectors in an a-b cross-section in this figure onto a vertical plane through this cross-section is shown in Fig.10(a). Here, the fish observed through this plane is presented. Since the 
center-line of the side jet is expected to be located at the mid-height of the body, probable streamlines are illustrated in the figure. Here, center of the vortex was adjusted to be between a region of upward fluid motion and a region of downward fluid motion. This is consistent with the observation in Fig.7(c) and supports the existence of the vortex ring surrounding the side jet.

After a period of $4 / 15 \mathrm{~s}$, the side jet is convected further away from the tail, and fish body is in a straight form, as shown in Fig.9(d). But suddenly, the fish bent its body again in Fig.9(e), and made a second turning motion by ejecting another side jet directed to the lower-right in Fig.9(f). This side jet accompanies counter-rotating vortices similar to, but larger than, the first one. At this time, the measurement plane is located nearly at the mid-height of the body as shown in Fig.9(f'). Since the velocity vectors in cross-section c-d, presented in Fig.10(b), are almost parallel to the horizontal plane, it is reasonable to understand that the vectors are of the center line of the side jet itself, as seen by the streamlines drawn in this figure. From Fig.9(a) to (d), the rotation angle of the body axis was 32 degrees, and from (d) to (e), the angle was 20 degrees. Thus the double turning achieves 52 degrees of rotation within a duration of $16 / 15 \mathrm{~s}$. In contrast to the single turning, the thrust jet was not produced behind the tail.

\subsection{Impulse and Propulsive Force}

The existence of the vortex ring surrounding the side jet leads us to estimate the impulse and time-averaged propulsive force acting on the fluid by employing the same procedure as that performed by Drucker and Lauder (1999). In this section, we estimate the momentum of the vortex ring, which is equivalent to the impulse acted by the tail fin, and timeaveraged force appeared in the fish motion shown in Fig.9. 
The momentum of a vortex ring (Milne-Thomson, 1966) is expressed as

$$
M=\rho \Gamma A
$$

where $\Gamma$ is a circulation along any closed loop surrounding a vortex line of the vortex ring, $A$ is the area surrounded by the vortex ring, and $\rho$ is the density of the fluid. When the tail fin swept and the isolated vortex ring was formed, an impulse $I$ acting on the fluid equals the momentum $M$, and the time-averaged propulsive force acting on the tail fin during the fin-stroke period $\Delta T$ is

$$
\bar{F}=\frac{\rho \Gamma A}{\Delta T} .
$$

We applied this procedure to the vortex rings shown in Fig.9(c) and (f). The circulation of the vortex ring was obtained by integrating the product of the velocity vectors and tangential unit vectors on a closed path surrounding the vortex core. The closed path was defined as a line of a constant vorticity $\omega_{z}=0.1 \omega_{z, \max }$, where $\omega_{z, \max }$ is the peak value of the $z$-component of vorticity of the vortex ring. The area of the ring was determined by $A=\pi D^{2} / 4$, where $D$ is the distance between the positive and negative vorticity peaks calculated from the velocity data shown in Fig.9(f), which ensures that the measurement plane is located closely in the center of the vortex ring as mentioned in Sec.3.2 with Fig.10(b). The ring diameter $D$ evaluated based on Fig.9(f) was $D=20.6 \mathrm{~mm}$, and was used to calculate the property for another vortex ring shown in Fig.9(c).

The properties of two vortex rings and their estimated forces are summarized in Table 1. Here, the duration of the fin-stroke was determined based on the images. However, it is a relatively small period comparing to the frame interval of the images. So the $\Delta T$ may have errors of approximately $50 \%$. Even so, the value of the force still have an order of magnitude the same as that of the vortex rings generated by the pectoral fins of the 
bluegill sunfish estimated by Drucker and Lauder (1999).

Note that the two vortex rings have approximately the same circulation and momentum. We perform rough estimation of the angular momentum of the fish body taking into account the surrounding fluid (added mass) in order to compare the momentum of the side jet. The height of the fish, $h(\xi)$, as a function of distance from the head toward the tail, $\xi$, was measured from a fish image viewed from the side. Then, the moment of inertia, $I_{m}$, of the fish body and the surrounding fluid was estimated assuming the crosssection of the body including the added mass was round in shape with a radius of $h / 2$. Here, the axis of the rotation was defined as a vertical line through the center of gravity. The calculated moment of inertia was $I_{m}=3.2 \times 10^{-5} \mathrm{kgm}^{2}$. Next, the angular velocity, $\Omega$, was assumed to be the total turning angles of the two successive turns divided by the time required to rotate that angle. As noted in the last paragraph of Sec.3.2, the total angle was 52 degrees and the time period was $16 / 15$ s. Thus $\Omega=0.85 \mathrm{rad} / \mathrm{s}$. Finally, the angular momentum was estimated as $L=I_{m} \Omega=2.7 \times 10^{-5} \mathrm{kgm}^{2} / \mathrm{s}$. This value is in the same order of magnitude as the total angular momentum of the two successive side jets, $L_{\text {total }}=(0.00048+0.00052) R=5.8 \times 10^{-5} \mathrm{kgm}^{2} / \mathrm{s}$, where $R$ is the distance between gravitational center of the body and the trailing edge of the tail. Although the above estimation is still ambiguous in the estimation of added mass and the angular velocity, it is consistent with the fact that the angular momentum was conserved by producing the side jet when the fish and its surrounding fluid rotate. 


\begin{tabular}{lcccccc} 
Name & Figure & $D(\mathrm{~mm})$ & $\Gamma\left(\mathrm{mm}^{2} / \mathrm{s}\right)$ & $M(\mathrm{Ns})$ & $\Delta T(\mathrm{~s})$ & $\bar{F}(\mathrm{mN})$ \\
\hline Vortex ring 1 & Fig.9(c) & $20.6^{*}$ & 1430 & 0.00048 & $2 / 15$ & 3.6 \\
Vortex ring 2 & Fig.9(f) & 20.6 & 1560 & 0.00052 & $2 / 15$ & 3.9
\end{tabular}

Table 1: Properties of vortex rings and estimated force.*Ring diameter was assumed to be same as that of Vortex ring 2 .

\section{Conclusion}

Stereoscopic PIV was applied to measure the three-component velocity field around a turning fish, in addition to the ordinary PIV measurement of two-component velocity in a horizontal plane at the mid-height of the fish. As well, the three-dimensional shape of the fish was characterized by the use of additional cameras in the case of stereoscopic PIV measurement.

The velocity field and body shape in a horizontal plane revealed by two-component PIV revealed the following turning-motion process. The fish bent its body into a $\mathrm{C}$ shape at the beginning of the turning motion. The body began to resume a straightened form by propagating the bending point backward, and produced a 'side jet' in the middle of counter-rotating vortices, which gave angular momentum to the fish body and surrounding added mass. Finally, the thrust jet was issued backward and the body moved forward.

Stereoscopic PIV data revealed both a side jet and thrust jet in the vertical plane behind the fish. The height of both jets was approximately the same as the height of the tail fin, and counter-rotating vortices were observed at the top and bottom of each jet. Three-component velocity vectors measured in a horizontal plane around a fish making successive double turns was consistent with the above observations. Conjunction with the 
data of both the horizontal plane and vertical plane gave evidence of the existence of the vortex ring around the side jet. Based on the circulation and diameter of the vortex ring, the impulse and time-averaged force in terms of the side jet were estimated.

\section{Acknowledgments}

We appreciate the help of the technical staff of our institute, Mr.T.Nakajima, Mr.M.Kobe and Mr.F.Yamada, for the construction of the calibration plate with three-dimensional traversing mechanisms. 


\section{References}

[1] Anderson JM; Streitlien K; Barrett DS; Triantafyllou MS (1998) Oscillating foils of high propulsive efficiency. J Fluid Mech 360: 41-72

[2] Breder CM (1926) The locomotion of fishes. Zoologica IV No.5: 159-297

[3] Drucker EG; Lauder GV (1999) Locomotor forces on a swimming fish: threedimensional vortex wake dynamics quantified using digital particle image velocimetry. J Exp Biology 202: 2393-2412

[4] Drucker EG; Lauder GV (2000) A hydrodynamic analysis of fish swimming speed: wake structure and locomotor force in slow and fast labriform swimmers. J Exp Biology 203: 2379-2393

[5] Drucker EG; Lauder GV (2001) Wake dynamics and fluid forces of turning maneuvers in sunfish. J Exp Biology 204: 431-442

[6] Gopalkrishnan R; Triantafyllou MS; Triantafyllou GS; Barrett D (1994) Active vorticity control in a shear flow using a flapping foil. J Fluid Mech 274: 1-21

[7] Gray J (1936) Studies in animal locomotion 6. The propulsive powers of the dolphin. J Exp Biol 13, No.2: 265-301

[8] Hecht E (1990) Optics (2nd ed.) pp.226: Addison Wesley

[9] Lighthill J (1960) Note on the swimming of slender fish. J Fluid Mech 9: 305-317

[10] Lighthill J (1970) Aquatic animal propulsion of high hydromechanical efficiency. J Fluid Mech 44: 265-301 
[11] Milne-Thomson LM (1966) Theoretical aerodynamics, New York: Macmillan

[12] Muller UK; Van Den Heuvel BLE; Stamhuis EJ; Videler JJ (1997) Fish foot prints: morphology and energetics of the wake behind a continuously swimming mullet (chelon labrosus risso). J Exp Biology 200: 2893-2906

[13] Prasad AK; Adrian RJ (1993) Stereoscopic particle image velocimetry applied to liquid flows. Exp Fluids 15: 49-60

[14] Solof SM; Adrian RJ; Liu ZC (1997) Distortion compensation for generalized stereoscopic particle image velocimetry. Meas Sci Tech 8: 1441-1454

[15] Stamhuis EJ; Videler JJ (1995) Quantitative flow analysis around aquatic animals using laser sheet particle image velocimetry. J Exp Biology 198: 283-294

[16] Taneda S; Tomonari Y (1974) An experiment on the flow around a waving plate. J Phys Soc Japan 36 No.6: 1683-1689

[17] Wolfgang MJ; Anderson JM; Grosenbaugh MA; Yue DKP; Triantafyllou MS (1999a) Near-body flow dynamics in swimming fish. J Exp Biology 202: 23032327

[18] Wolfgang MJ; Triantafyllou MS; Yue DKP (1999b) Visualization of complex near-body transport process in flexible-body propulsion, J Visualization 2 No.2:143151

[19] Wu TYT (1971) Hydrodynamics of swimming propulsion. Part 2. Some optimum shape problems. J Fluid Mech 46: 521-544 
Figure 1: Arrangement of the water tank and instrumentation.

Figure 2: Schematic diagram of the physical particle location and its location on the image planes.

Figure 3: Intersection of two lines.

Figure 4: Schematic diagram of the physical location of the fish and its projection to the cameras placed at the bottom and to the side.

Figure 5: Fish body projected from side (a) and bottom (b). Performing a logical-AND operation between (a) and (b), a 3D-shape of the fish can be estimated as (c).

Figure 6: Velocity vectors around a turning fish. The iso-vorticity contours are overlaid with a solid line for the positive value and a gray line for the negative value. Vectors above the fish were deleted since the light sheet was shadowed by the fish body. (a)t $=$ $0 \mathrm{~s} ;(\mathrm{b}) t=1 / 15 \mathrm{~s} ;(\mathrm{c}) t=3 / 15 \mathrm{~s} ;(\mathrm{d}) t=8 / 15 \mathrm{~s}$.

Figure 7: Velocity vectors behind a turning fish. Vectors in the right column represent the $v$ and $w$ components of velocity, and the intensity of the red (blue) color represents the magnitude of the negative (positive) $u$ component of velocity. The right column shows data identical to that in the left column but viewed from the top, and the $u$ and $v$ components of the velocity vectors are shown. Tic intervals were $10 \mathrm{~mm}$. (a)t $=0 \mathrm{~s}$; (b) $t=7 / 15 \mathrm{~s} ;(\mathrm{c}) t=16 / 15 \mathrm{~s} ;(\mathrm{d}) t=22 / 15 \mathrm{~s}$. 
Figure 8: Schematic drawing of the arrangement of the vortex ring surrounding the side jet as the fish makes a turn.

Figure 9: Velocity vectors around a fish which make two successive turns. The vecotrs represent $u$ and $v$ components, and the intensity of the red (blue) color represents the magnitude of the negative (positive) $w$ component of velocity. (a) $t=0 \mathrm{~s}$; (b) $t=2 / 15 \mathrm{~s}$; (c) $t=5 / 15 \mathrm{~s} ;(\mathrm{d}) t=9 / 15 \mathrm{~s} ;(\mathrm{e}) t=12 / 15 \mathrm{~s} ;(\mathrm{f}) t=16 / 15 \mathrm{~s} .\left(\mathrm{c}^{\prime}\right)$ and $\left(\mathrm{f}^{\prime}\right)$ are respectively the same data as (c) and (f), but viewd from the back of the fish. Lines a-b and c-d in (c) and (f) represent the location of vectors shown in Fig.10.

Figure 10: Velocity vectors on lines a-b and c-d in Fig.9. 


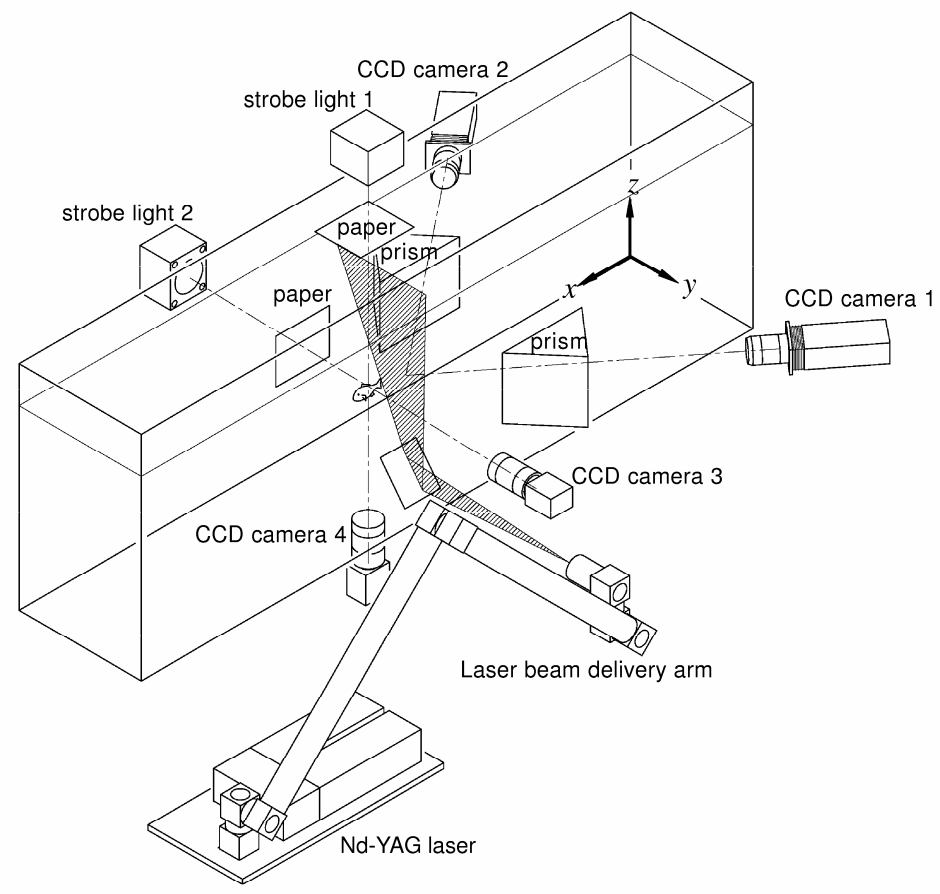

Fig. 1 


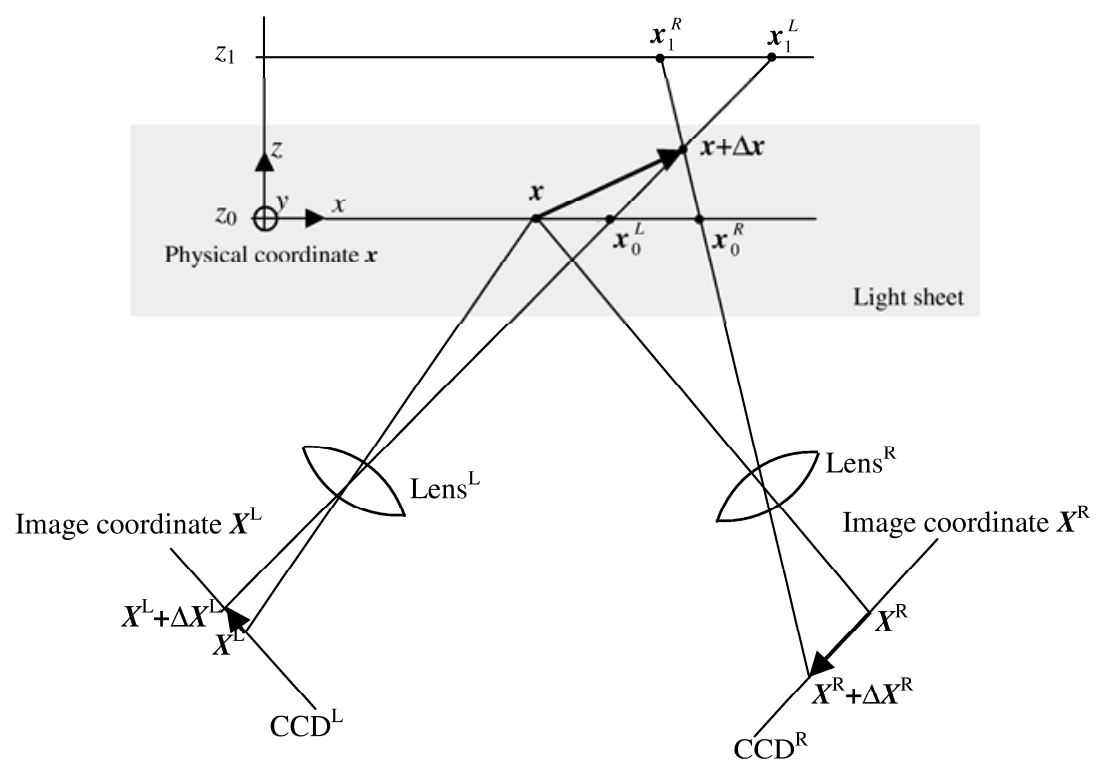

Fig.2 


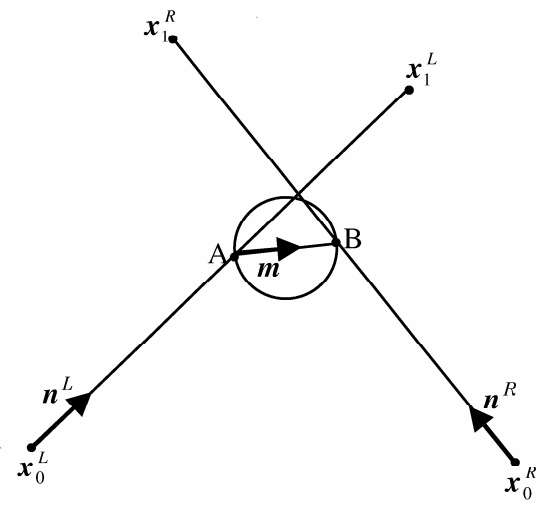

Fig.3 


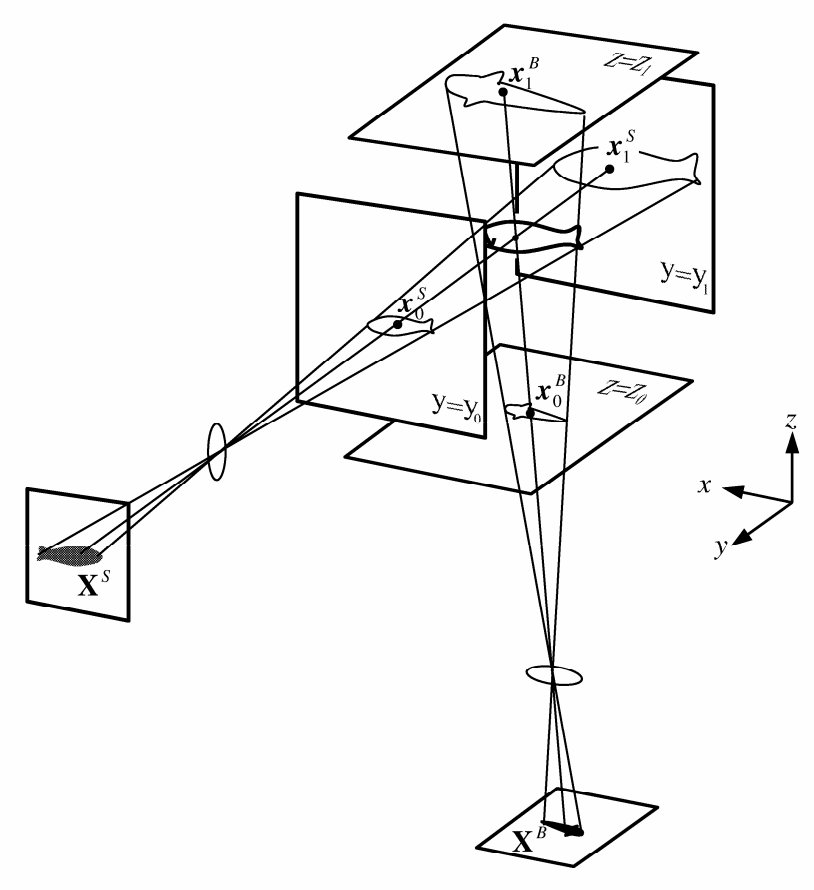

Fig.4 

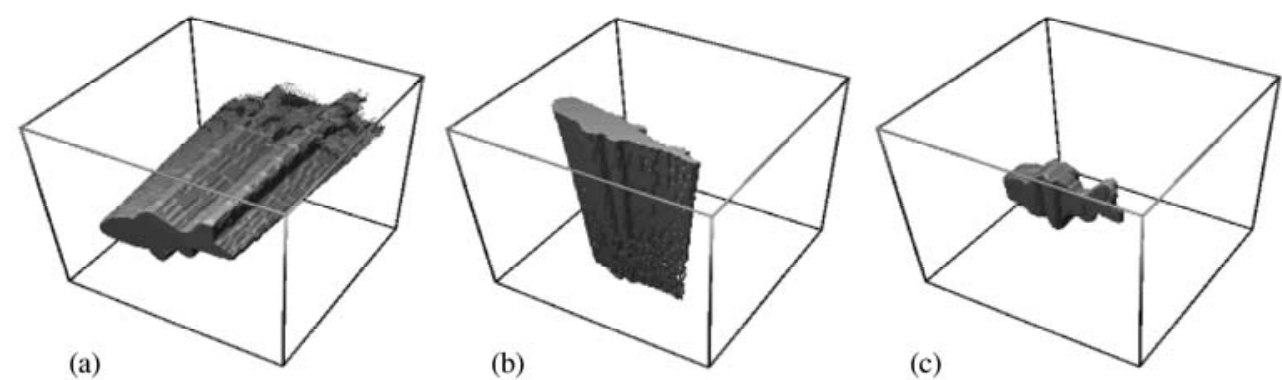

Fig. 5 
(a)

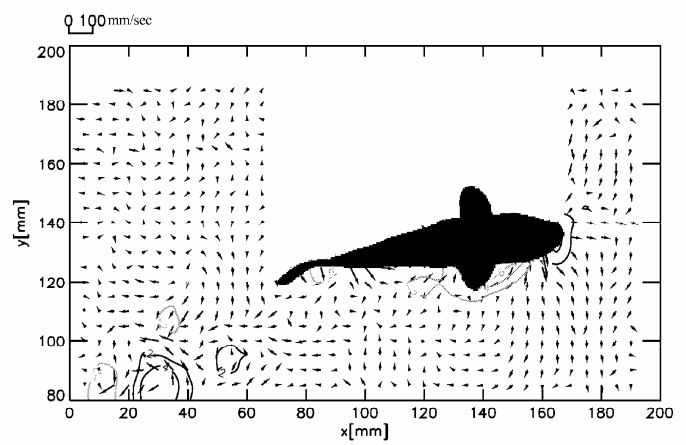

(b)

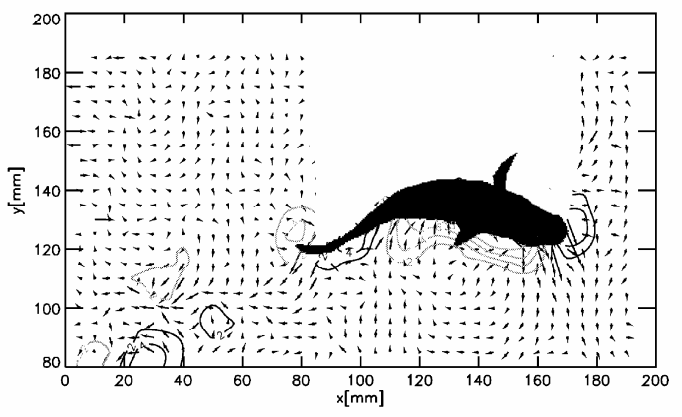

(c)

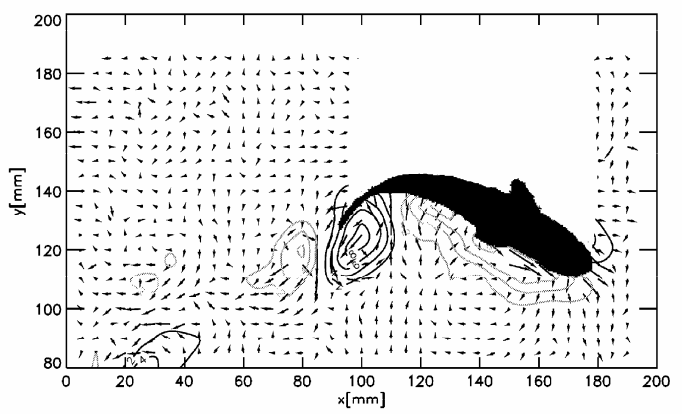

(d)

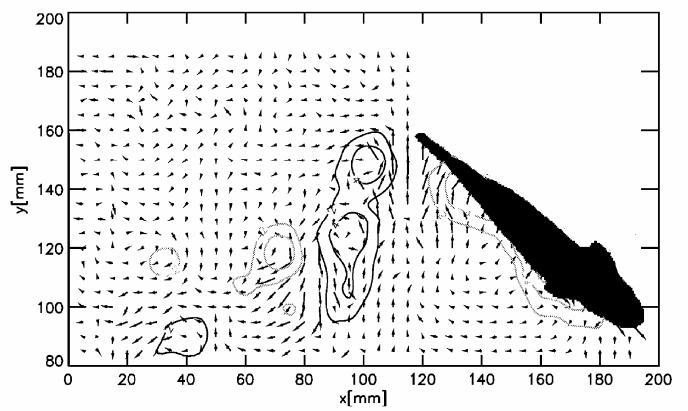

Fig.6 
(a)

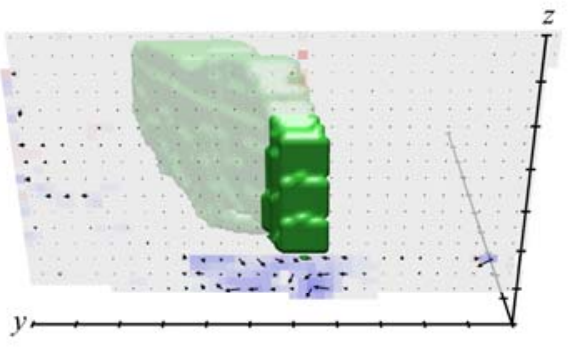

(b)

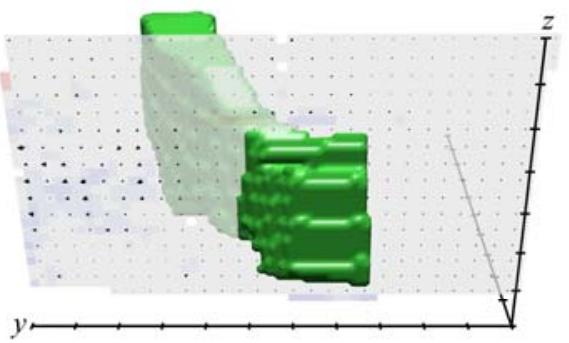

(c)

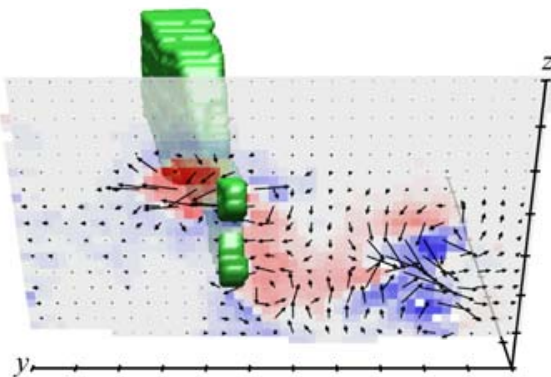

(d)

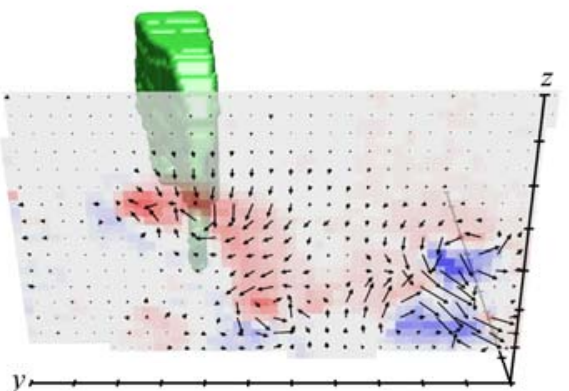

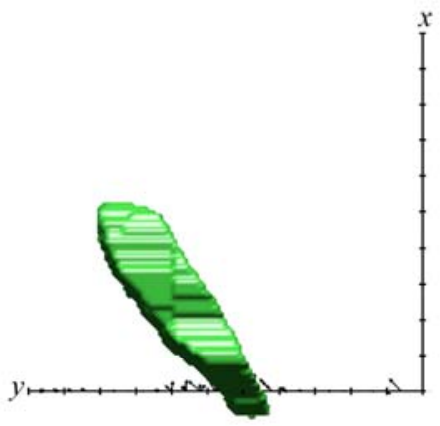
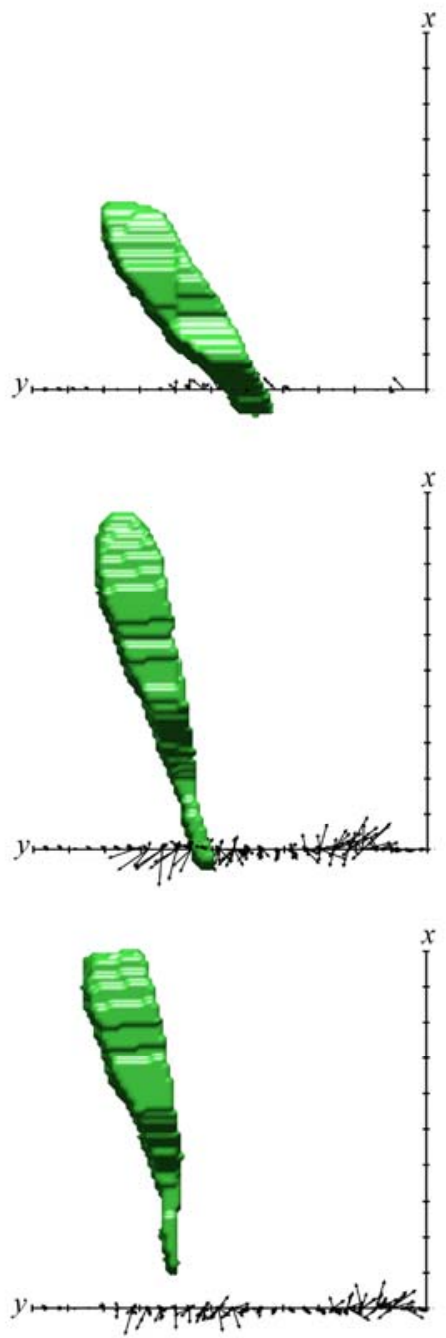

Fig.7 


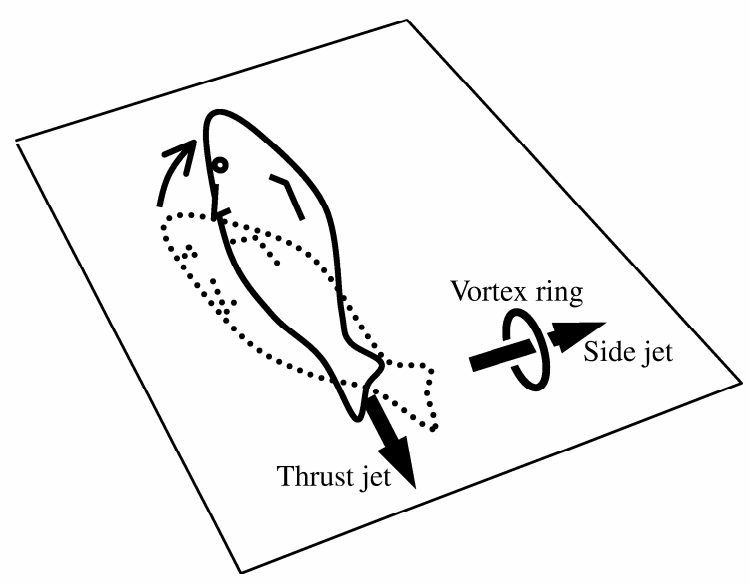

Fig. 8 

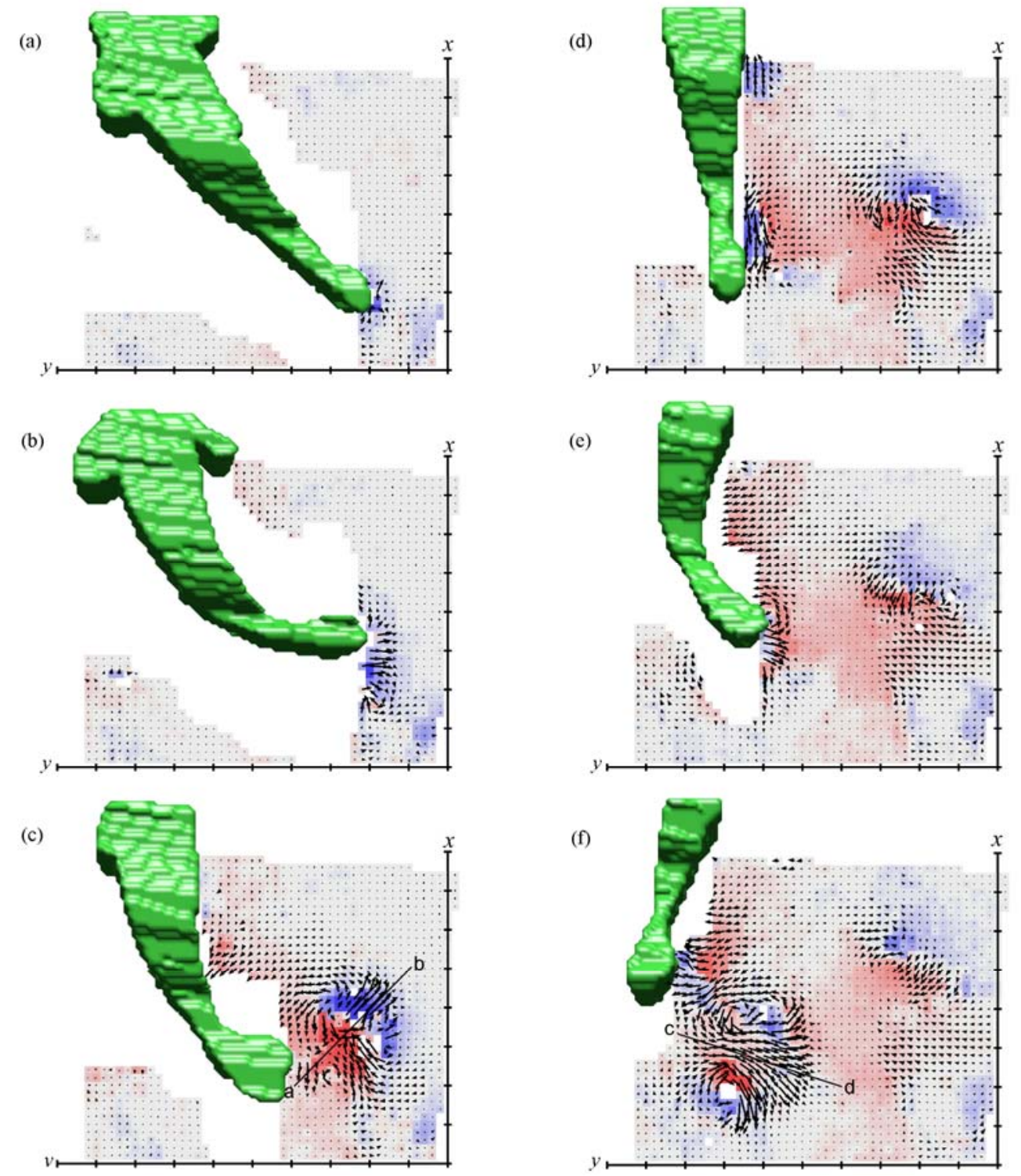

(c')

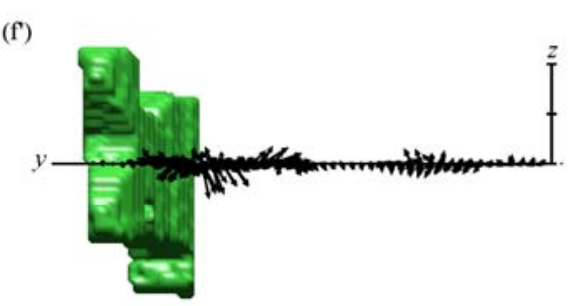

Fig.9 

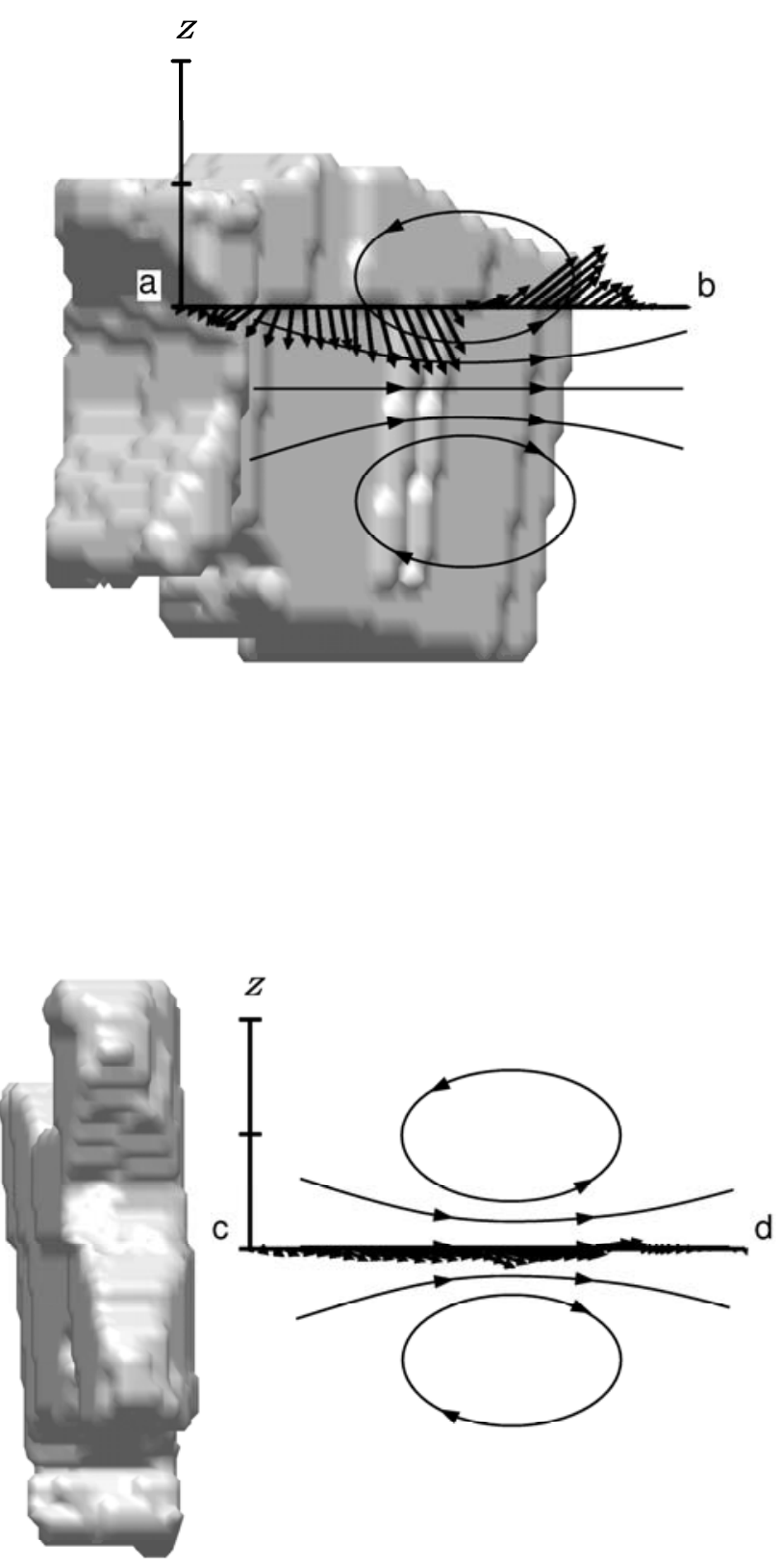

Fig.10 